\title{
Emergence of Global Network Property based on Multi-agent Voting Model
}

\author{
Kosuke Shinoda \\ National Defense Academy \\ 1-10-20 Hashirimizu, \\ Yokosuke \\ Kanagawa, Japan \\ kshinoda@nda.ac.jp
}

\author{
Yutaka Matsuo \\ National Institute of Advanced \\ Induastrial Science and \\ Technology \\ 1-18-13 Sotokannda, Chiyoda \\ Tokyo, Japan \\ y.matsuo@aist.go.jp
}

\author{
Hideyuki Nakashima \\ Future University, Hakodate \\ 1 \\ Hokkaido, Japan \\ h.nakashima@fun.ac.jp
}

\begin{abstract}
Recent studies have shown that various models can explain the emergence of complex networks, such as scale-free and small-world networks. This paper presents a different model to generate complex networks using a multi-agent approach. Each node is considered as an agent. Based on voting by all agents, edges are added repeatedly. We use four different kinds of centrality measures as a utility functions for agents. Depending on the centrality measure, the resultant networks differ considerably: typically closeness centrality generates a scale-free network, degree centrality produces a random graph, betweenness centrality favors a regular graph, and eigenvector centrality brings a complete subgraph. The importance of the network structure among agents is widely noted in the multi-agent research literature. This paper contributes new insights into the connection between agents' local behavior and the global property of the network structure. We describe a detailed analysis on why these structures emerge, and present a discussion of the possible expansion and application of the model.
\end{abstract}

\section{Categories and Subject Descriptors}

I.2 [Artificial Intelligence]: Distributed Artificial Intelligence

\section{General Terms}

Design, Experimentation

\section{INTRODUCTION}

Social networks are received much attention in the multiagent research community as well as other research domains such as Web technology [17], ubiquitous technology [14] and social science [21]. Recently, social networking services (SNSs) have become popular on the Web. Each user registers their

Permission to make digital or hard copies of all or part of this work for personal or classroom use is granted without fee provided that copies are not made or distributed for profit or commercial advantage and that copies bear this notice and the full citation on the first page. To copy otherwise, to republish, to post on servers or to redistribute to lists, requires prior specific permission and/or a fee.

WOODSTOCK' 97 El Paso, Texas USA

Copyright 200X ACM X-XXXXX-XX-X/XX/XX ...\$5.00. acquaintances, thereby demarcating a large social network. Various applications use social networks, e.g., information recommendation [13] and information sharing [12]. The SNSs are considered as multi-agent systems: each agent (or a user) interacts locally, registering friends and acquaintances, exchanging information by writing and commenting on weblogs (blogs). Several studies have regarded social network as a multi-agent system and proposed applications such as information recommendation [22] and publication search [4].

Despite the importance of social networks for multi-agent systems, few studies have been conducted into the nature of local behavior and global network properties. Some studies have revealed that the overall performance is markedly influenced by the network structure among agents $[2,8]$. However, if we examine the application to social network systems such as information recommendation, it is necessary to have knowledge about how local behavior will produce the overall network structure, rather than how the performance differs depending on a given network structure.

In this paper, we propose a growing network model from the multi-agent point of view, which is intended to show the insight between the local behavior of the agents and the resultant network structure. In our model, each agent (recognized as a node) attempts to increase their utility by adding new edges. The addition of an edge might be preferred by some agents, but not by other agents. Resultant conflicts among agents can be resolved using a voting system. This situation is understood and justified by several social network examples in the real world: For example, in a large organization, some people selects others to join their "clique", and thereby strengthen it. In political organizations and academic organizations, people sometimes try to grow their own groups. They encourage others to be more connected to their group. Therefore, it is important to investigate network properties based on an agreement among multiple agents.

Our problem setting is as follows: each agent is considered as a node. A new edge is generated through agreements among agents, as recorded through a vote. The newly invented edge increases the respective utilities of some agents. This process is iterated and the network becomes more connected. We use centrality measures for a utility functions. Centrality, which represents how "central" an actor is in a social network, has been long studied in the field of social network analysis. Especially, we select four centrality measures, degree, closeness, betweenness and PageRank central- 
ity, and show that the selection of centrality measure yield a significant difference in global network properties.

The contribution of the paper can be summarized as follows:

- We show how local interaction and agreement among agents consists different network properties. Our model is simple; for that reaseon, we can show the basic phenomenon involving local behaviors and global properties.

- Our results are useful in the design of social network services by a multi-agent system, especially in relation to recommendations of information, people, and communities. We show the preliminary analysis of an actual social network, and discuss the applicability of our findings for a recommendation system.

The paper is organized as follows. In the next section, we describe related studies. Then we explain the algorithm of our simulation in more detail. Section 4 and 5 are devoted to results of simulation and theoretical analysis on the results. Section 6 discusses the expansion and applicability of the model, and we conclude the paper.

\section{RELATED WORKS}

Some studies have investigated interactions among agents and system behavior at the collective level [8, 2]. Axtell shows the effects of distinct agent interaction and overall structures in retirement behavior and firm formation. Gaston demonstrated that the social network structure underlying an agent organization can have considerable impact on organizational performance. In these studies, a network model is given such as a lattice, a random graph, or a small-world graph. Although the motivation is similar toward the importance of network structure among agents, the approaches of those studies and ours are different, or strengthen each other: it is important not only to know the relation between a certain network structure and the overall performance, but also the relation between local behavior and network structures.

Mechanism of network generation such as scale-free or small world networks are studied in the context of complex networks $[3,20]$. The most popular explanation of scale-free networks is preferential attachment: A newly created node is connected to a pre-existing one with a probability proportional to the number of edges of the target node. Other models include connecting nearest-neighbor model and mutual selection model [18], both of which explain the emergence of scale-free networks. In the latter model, a new edge is added based on mutual affinity between nodes, which can be considered as a simple multi-agent system. However, most studies do not employ the multi-agent system: they do not explicitly have agents' decision, goals or utility functions.

A similar study to ours is that of network games, which investigates the dynamic formation and evolution of networks. Jackson and Watts proposed a model [10] in which all agents have the same value function and in which new edges are added by agreement between two agents when they meet randomly. Our model is distinguished from the one in that study because we employ a voting system as a global agreement mechanism; moreover, we clarify the relation to network structures such as a scale-free or a random graph.
Social networks are important for various multi-agent systems. In the previous section, we described studies using social networks for information recommendations and distributed searches [22, 4]. Another major application is trust and reputation calculation: In the Regret system proposed by Sabater [16], a reputation is calculated based on social network analyses. Pujol et al. develop a method to calculate reputation using only local information. It can be adapted adapted to the network topology [15]. Ashri et al. proposes a method to evaluate the trustworthiness of agent's counterparts, based on an analyses of relationships [1]. Our study can contribute to these research efforts by generating appropriate social networks depending on their intended purposes.

\section{SIMULATION MODEL}

In this section, we first overview centrality measures, which are studied in sociology. The centrality measure is used to represent the utility of an agent in our model. The overall setting of the simulation is explained next. In our model, nodes are considered as agents, and edges are considered as relations between agents, which are non-directional and reciprocal.

\subsection{Centrality as Utility}

Social network analysis includes various measures of node centrality that determine the relative importance of a node within the network [19]. It represents the structural importance of a node, which can perhaps be characterized as the power of individual actors. Among the several kinds of centrality measures [7], the most popular ones are degree, closeness, betweenness, and eigenvector centrality.

In our model, we assume that each agent seeks to increase its centrality. In other words, centrality is used as a proxy for utility, which is to be maximized. Our intuition is simple: recognizing that traditional studies in social science have shown the usefulness of centrality as a measure of power, why not infer it as a proxy for utility? In other words, we assume that an agent behaves to increase its power that can be calculated by a centrality measure.

In our model, we use four kinds of centrality measure:

Degree The degree of a node is the number of edges to other nodes. Actors who have more ties to other actors might be in and advantageous positions. It is defined as $C_{i}^{D}=\frac{k_{i}}{N-1}$ where $k_{i}$ is the degree of node $i$ and $N$ is the number of all nodes.

Closeness Closeness centrality captures how close an actor is to all the other actors. It is calculated by the minimum distance of an actor to all other nodes. Unlike other centrality measures, the smaller the value is, the more central the actor. It is defined as $C_{i}^{C}=\left(L_{i}\right)^{-1}=$ $\frac{N-1}{\sum_{j \in G} d_{i j}}$ where $L_{i}$ is the average geodesic distance of node $i$, and $d_{i j}$ is the distance between node $i$ and $j$. To facilitate calculation for disconnected components, we set $d_{i j}=N$ if nodes $i$ and $j$ are disconnected.

Betweenness Th betweenness centrality measures indicates that an actor is in a favored position if the actor falls on the shortest paths between other pairs of actors in the network. It measures the number of all the shortest paths that go through the node, and is defined as the following. $C_{i}^{B}=\sum_{j, k \in N} n_{j k}(i) / n_{j k}$ In that equation, 
(d)<smiles>[CH2][CH-][CH2-]</smiles>
(e)

[1]

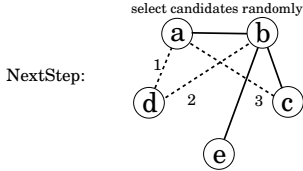

[5]

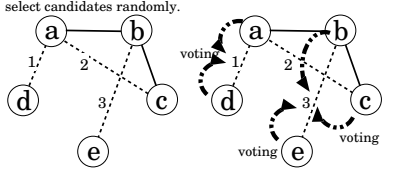

[3]

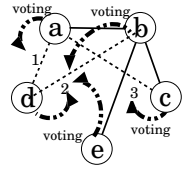

[6]

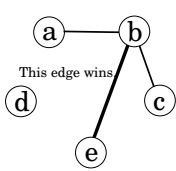

[4]

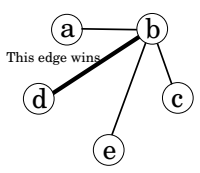

[7]
Figure 1: Example of network growth.

$n_{j k}$ denotes the number of the shortest paths between node $j$ and $k$, and $n_{j k}(i)$ is the number of those running through node $i$. ${ }^{1}$

PageRank PageRank [6] was proposed as a measure of the importance of a Web page. Although PageRank corresponds to eigenvector centrality (under a proper transformation), we use PageRank because of its familiarity to computer science researchers. It is calculated iteratively as the following. $C_{i}^{P}(t+1)=\alpha A_{i}+(1-$ $\alpha) \sum_{j \in B_{i}} C_{j}^{P}(t) / D_{j}$ Therein, $D_{j}$ is the degree of node $j, B_{i}$ is the number of neighbors of node $i, A_{i}$ is a vector representing the source of the network. We set $\alpha=0.15$ and $A_{i}$ as uniform: each element of $A_{i}$ is $1 / N$. This calculation is iterated until $C^{P}$ converges.

\subsection{Simulation Setting}

We first explain the motivation of our simulation model using a couple of examples. The first example is highway construction among cities. Assume that the cities initially have no highways. Resident of either city would be happy if a highway is constructed between two cities. The next highway might be constructed anywhere else, but every city wants it because it improve the closeness centrality, i.e., citizens gain convenience. Consequently, a possible solution is that they decide the issue through negotiation or voting. Several candidates exist for the construction of highway; every city vote for one of them. The winner performs the actual construction. In this manner, cities gradually become more connected. A possible result might be an emergence of a hub city: every citizen travel to other cities in a short time if there is such a hub city.

We can take another example in a social network in an organization or a group. A person would like to be position between people to thereby maintain control of information flow, i.e. to increase betweenness centrality. In this situation, the resultant network is less likely to have hubs because people would compete to hold that central position.

Based on such intuitions, we design our simulation in the following manner. Each agent is separated at first. The candidate edges are selected next. Every agent has preferences on the addition of an edge, because addition of an edge would increase or decrease the agent's utility, i.e., a centrality measure. Each agent votes for the candidate edges, and the edge with the most votes is eventually added. This repeated process renders the network as increasingly connected.

\footnotetext{
${ }^{1}$ In our simulation, we use the fast algorithm to calculate the betweenness [5].
}

\section{Initialize}

- Assume $N$ nodes. Set the number of candidates as $c$.

2. Choose candidates

- Select a node randomly. Among the possible edges from the node, select one randomly as a candidate. Repeat until the number of candidates is $c$, or until there are no possible edges.

3. Evaluate candidates and vote

- Each node evaluates each candidate using the utility function $f$.

- Score the candidates in decreasing order as $c-1$, $c-2, \ldots, 0$. If multiple candidates exist with the same utility, order them randomly.

4. Add an edge

- Add an edge corresponding to the candidate with the highest score.

\section{Terminate}

- Terminate if the number of edges greater than a given number, or if no possible edges exists. Otherwise repeat the iteration and go back to 2 .

Figure 2: Simulation procedure.

An illustration is presented in Figure 1. Initially, we have five nodes and two edges, and three candidate edges which are chosen randomly from possible edges. Each agent votes for one candidate. In this case, Edge 1 obtains two votes, Edge 2 obtains zero, and Edge 3 obtains three votes. Consequently, Edge 3 is added. Then we have another three candidates, and one of them is going to be built.

Details of the simulation procedure are shown in Figure 2. Several points are required for consideration:

How to select candidates There are several ways to choose candidate edges. We employ a simple one: we select edges randomly from the possible new edges. We use a parameter $c$, which denotes the number of candidates. If $c$ is large, the edge will be chosen among many possible edges; if $c$ is 1 , the procedure is completely random.

Score of candidates Each agent evaluate the candidate using a given centrality measure (one of degree, closeness, betweenness, and PageRank), and vote to them. We assume that all the agents use the same centrality to see how different network would appear.

Voting Various ways exist for voting. As a voting method, we adopt Borda counting, as devised by Jean-Charles de Borda. The method is used for single-seat or multipleseat elections. Because it sometimes elects broadly acceptable candidates, the Borda count is often described as a consensus-based electoral system, Each agent puts $c-1$ points to the first choice, $c-2$ points to the second choice, and 0 point for the least favorable choice. The candidate with the highest score wins.

\section{RESULTS AND OVERALL PROPERTY}

In this section, we describe results of network generation simulation. Especially, we examine differences among the four centrality measures. 

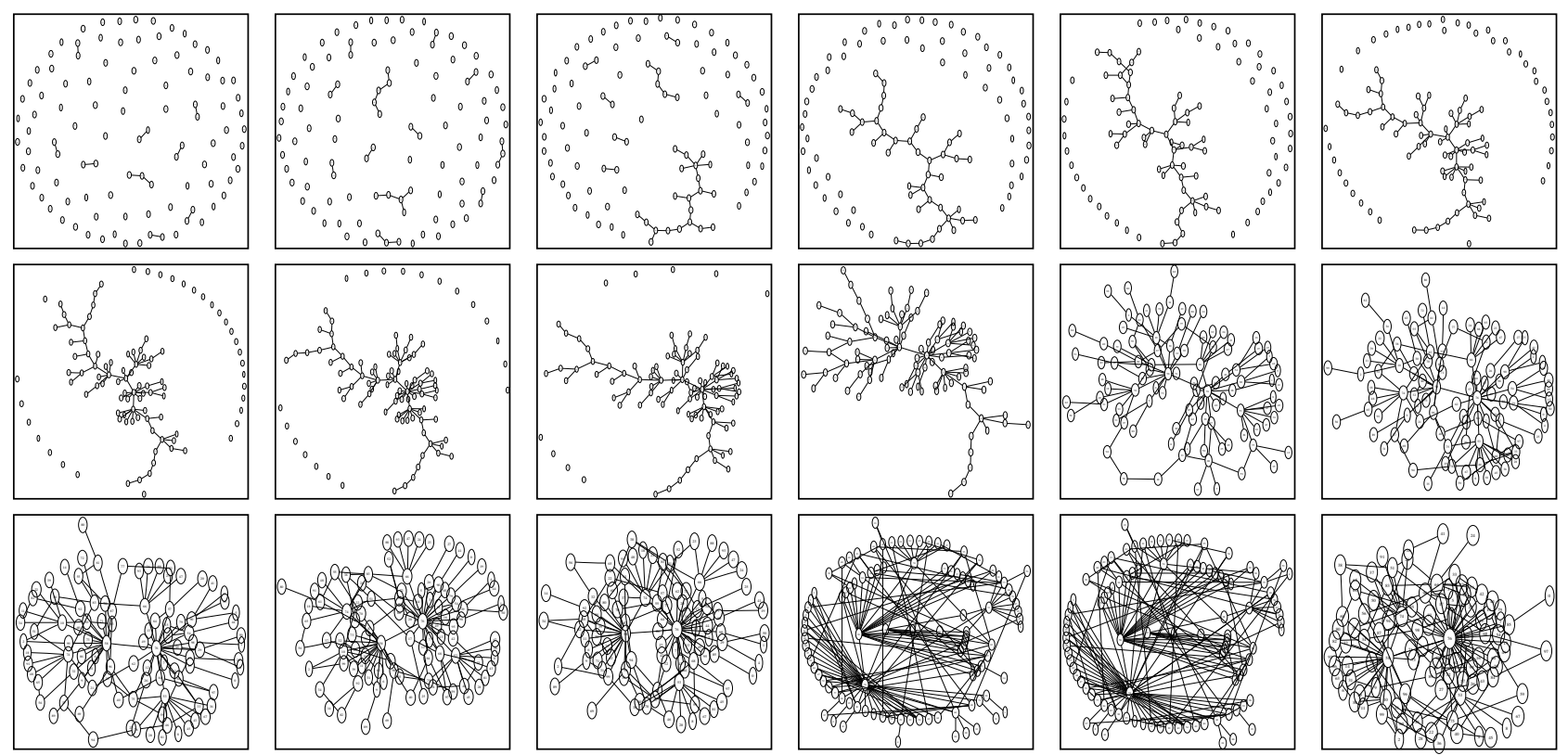

Figure 3: Network growth using closeness centrality as a utility. $N=100, c=200$. Up to 180 edges.

\subsection{Closeness Centrality}

Figure 3 shows how a network grows when using closeness centrality as agents' utility. We set the number of nodes $N$ to 100 and the number of candidates $c$ to 200. In each network, ten edges are newly added. Note that nodes are positioned for visualization. For that reason, the position and the distance of nodes imply nothing ${ }^{2}$.

Initially, the network has $N$ nodes without edges. When we add edges, several small components are generated first, which are subsequently connected together, thereby creating a "big island" (when the number of edges $K$ is 40 ). The large component gradually gets larger connecting other isolated nodes. Finally, all nodes are connected (when $K=100$ ). We can see two nodes with high degree. These are called hubs. Subsequently, nodes becomes gradually more directly connected to either of the two hubs. When $K=180$, almost every node is connected through the two hubs.

If we decrease $c$, the edge selection becomes more random. It therefor becomes unlikely that a hub will emerge. If we increase $c$, the network will show one or two hubs. Figure 4 shows the degree distribution of the generated network. We see the power-law distribution in the case of closeness centrality (Figure 4(a)) because it forms a straight line on a logarithmic-linear plot. Characteristically, these networks have "mega-hub", i.e. a node with huge degree.

Figure 1 shows some properties of generated networks. Regarding closeness, the characteristic path length $L$ is the smallest among them. The clustering coefficient $C$ is as low as a random graph. As $c$ becomes larger, $L$ gets smaller, which means a larger hub(s) appears.

\subsection{Betweenness Centrality}

Figure 5 shows network growth using betweenness centrality. Initially, the network grows similarly to that of closeness centrality, until the giant component includes all the nodes at $K=100$. Afterward, the edges connecting two nodes in a small distance are connected. Typically, there appears a network with edges connecting only neighbors, as shown in

\footnotetext{
${ }^{2}$ We use spring-embedded model for a network drawing.
}

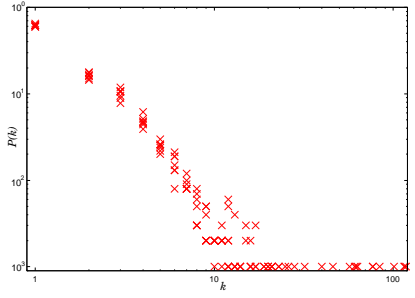

(a) Closeness

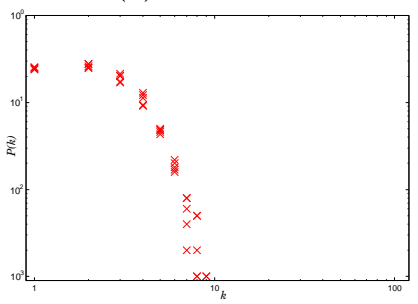

(c) Degree

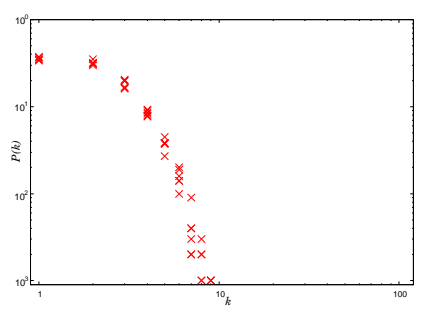

(b) Betweenness

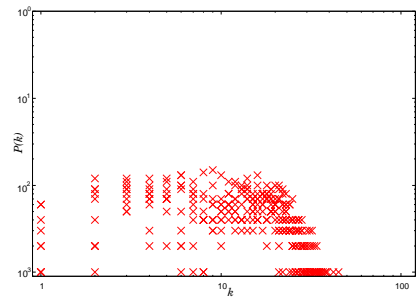

(d) PageRank
Figure 4: Degree distribution of network ( $N=1000$, $c=\mathbf{1 0 0 0}\rangle k,\langle=\mathbf{2 . 1})$

\section{Figure 6.}

The reason is understood to be the following: for each agent to increase betweenness centrality, $L$ should be large. Large $L$ indicates long shortest paths. Consequently, each node tends to be included in the shortest paths. We can see that $L$ is large as shown in Table 1 compared to other networks. If $c$ gets larger, $L$ gets larger too. The degree distribution of network is shown in Figure 4(b): Most nodes have degree of 2; even the largest degree is smaller than 10.

\subsection{Degree Centrality}

Figure 7 illustrates the network growth using degree centrality. Initially, there appear several pairs of nodes; they are gradually connected. However, the network does not show hubs or string-type growth. Isolated nodes are not easily connected to the largest component. Therefore, there are several isolated nodes even at $K=150$. 

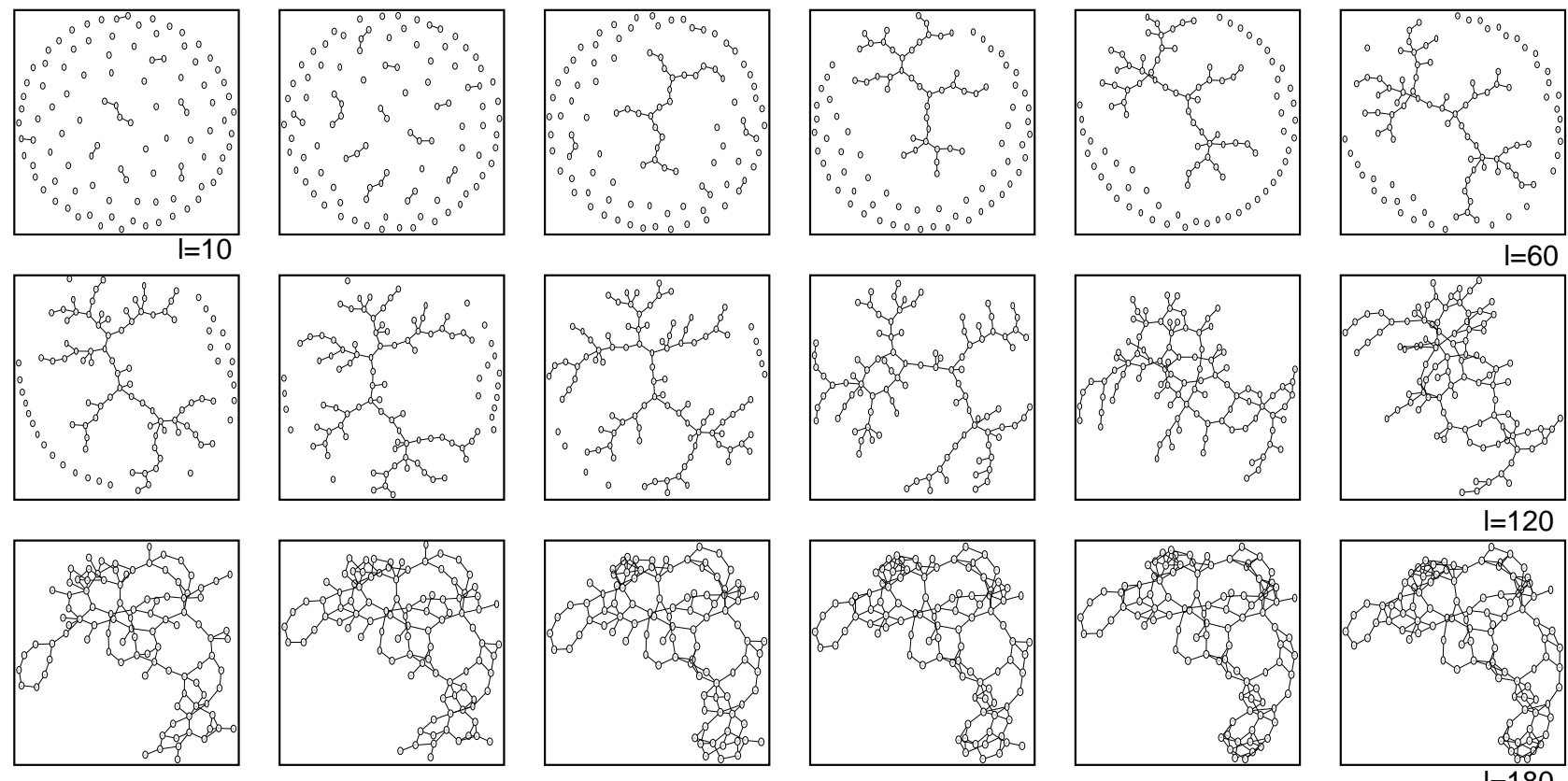

Figure 5: Network growth using betweenness centralitv. $N=100 . c=200$. Un to 180 edges.

Table 1: $L$ and $C$ for different $c$ values.

\begin{tabular}{l|l|l|l} 
centrality & $c$ & L & C \\
\hline Closeness & 20 & $4.54(4.72)$ & $0.000655(0.00857)$ \\
Closeness & 100 & $3.54(4.54)$ & $0.00898(0.00766)$ \\
Closeness & 200 & $3.32(3.32)$ & $0.00765(0.00582)$ \\
Closeness & $\max$ & $2.49(2.49)$ & $0.0212(0.0212)$ \\
\hline Betweenness & 20 & $8.28(8.46)$ & $0.00565(0.00564)$ \\
Betweenness & 100 & $8.45(8.62)$ & $0.00552(0.00381)$ \\
Betweenness & 200 & $8.54(8.54)$ & $0.00796(0.00796)$ \\
Betweenness & $\max$ & $9.97(9.97)$ & $0.0113(0.0113)$ \\
\hline Degree & 20 & $5.50(38.8)$ & $0.0117(0.0103)$ \\
Degree & 100 & $5.45(35.2)$ & $0.0166(0.0144)$ \\
Degree & 200 & $5.19(36.2)$ & $0.0140(0.0123)$ \\
Degree & $\max$ & $4.85(35.6)$ & $0.0170(0.0149)$ \\
\hline PageRank & 20 & $2.27(86.3)$ & $0.201(0.0751)$ \\
PageRank & 100 & $1.82(93.7)$ & $0.425(0.109)$ \\
PageRank & 200 & $1.69(96.1)$ & $0.593(0.131)$ \\
PageRank & $\max$ & $1.50(96.14)$ & $0.866(0.175)$
\end{tabular}

$N=100, K=210$. We show $L$ and $C$ with isolated nodes and without isolated nodes in the parentheses.

In case of degree centrality, the addition of a new edge effects only the degree centrality of nodes at both ends. It has no global effects: Each candidate edge obtains solid votes only from the both ends, Other nodes will vote randomly. Thus the resultant network is a random graph. The degree distribution of network is shown in Figure 4(c). It follows Poisson distribution with mode value of 2 . Table 1 shows that $C$ and $L$ are small, which is also the characteristics of a random graph.

\subsection{PageRank}

Figure 8 illustrates the network growth using PageRank. Most nodes are isolated even if we add a sufficient number of edges; About $70 \%$ of the nodes are isolated at $K=150$. Only a fraction of the nodes are connected. These nodes are highly connected, consisting almost a complete graph.

If we look at Table $1, L$ is very large if we include the isolated nodes. However, $L$ within the connected components is as low as 1.5-2.0. $C$ is very large compared to other networks. The degree distribution in Figure 4(d) shows the
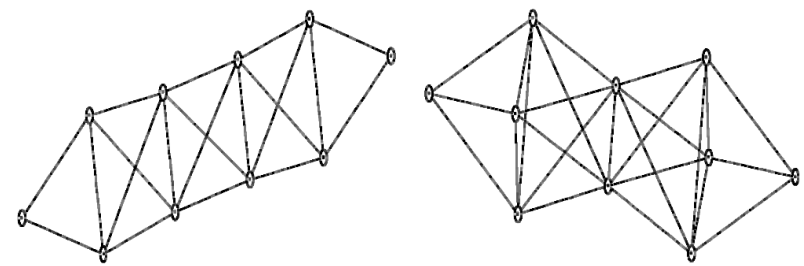

Figure 6: A typical case using betweenness centrality. $N=10, c=45, K=20$ (right-side) and $K=25$ (leftside).

(g)

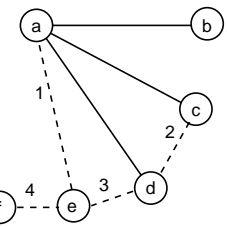

Figure 9: Illustration of edge addition in case of closeness centrality.

uniform distribution on degrees.

\section{MECHANISM OF NETWORK GROWTH}

In this section, we investigate network growth more theoretically. We explain how and why network of different types are emerged. We omit the explanation of degree centrality here because the resultant network is random. For the reason, it is easy to understand the mechanism.

\subsection{Closeness Centrality}

The network using closeness centrality is characterized by the emergence of hubs. We consider the mechanism of the hub appearance by showing that newly added edges strengthen the hub when given a hub.

Figure 9 exemplifies the situation where a hub is going to be generated: the network has three edges among seven nodes. Node a has three edges and is a small hub. Assume that four edges (as shown in dotted lines) are selected as 

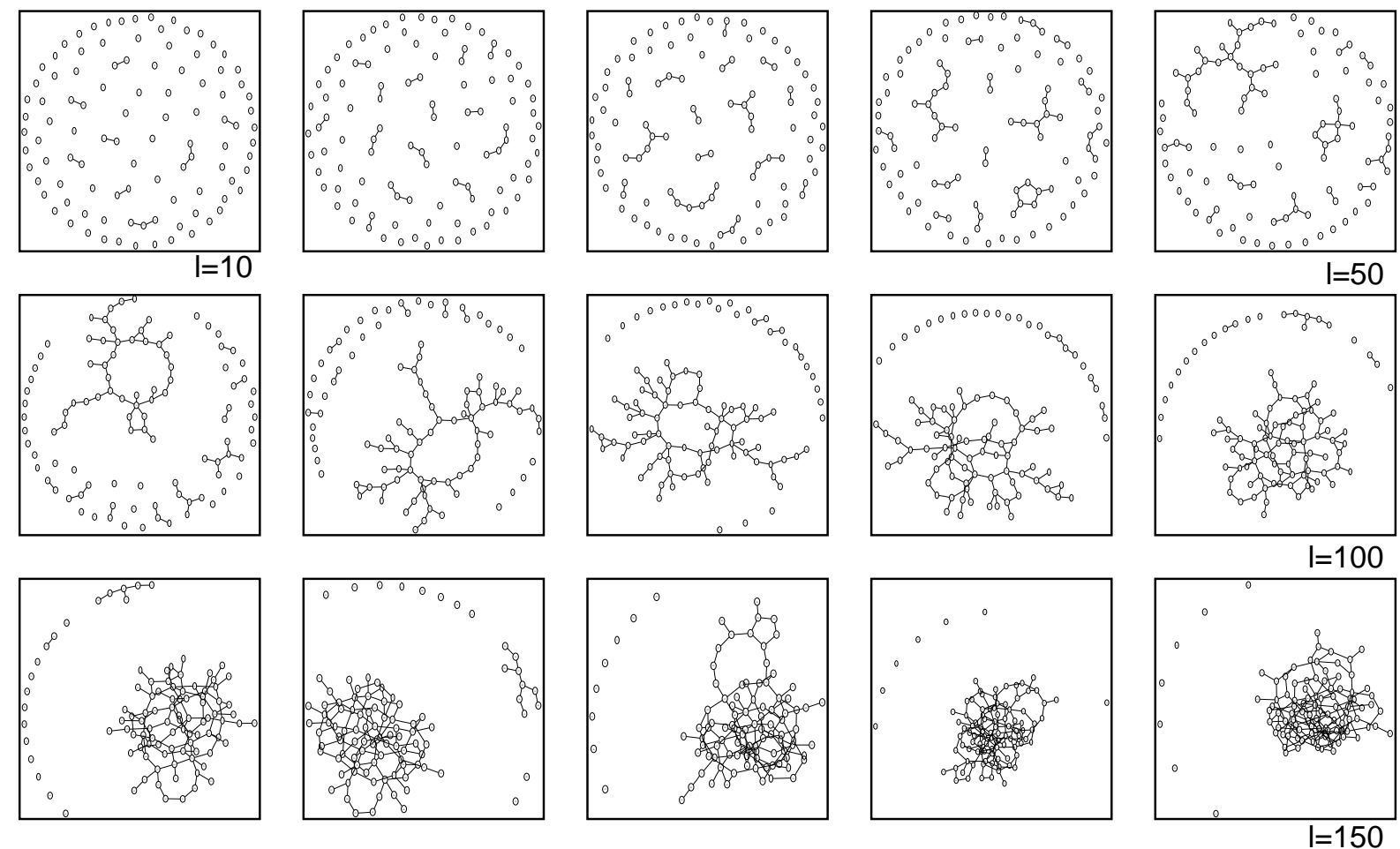

Figure 7: Network growth using degree centrality. $N=100, c=200$. Up to 150 edges.

Table 2: Expected number of votes in case of closeness.

\begin{tabular}{l|ccccccc|c} 
& $\mathrm{a}$ & $\mathrm{b}$ & $\mathrm{c}$ & $\mathrm{d}$ & $\mathrm{e}$ & $\mathrm{f}$ & $\mathrm{g}$ & $\mathrm{Sum}$ \\
\hline Edge 1 & 3 & 3 & 3 & 2 & 3 & 1 & 1.5 & 16.5 \\
Edge 2 & 0.5 & 0.5 & 0.5 & 0.5 & 0 & 1 & 1.5 & 4.5 \\
Edge 3 & 2 & 2 & 2 & 3 & 2 & 1 & 1.5 & 13.5 \\
Edge 4 & 0.5 & 0.5 & 0.5 & 0.5 & 1 & 3 & 1.5 & 7.5 \\
\hline
\end{tabular}

candidates. We denote a node that links to a hub such as Node b, c, d as a peripheral node. These can be four edge categories, which can be described as follow:

Edge 1 an edge which connects a hub and an isolated node,

Edge 2 an edge which connects two peripheral nodes,

Edge 3 an edge which connects a peripheral node and an isolated node, and

Edge 4 an edge which connects two isolated nodes.

The expected number of votes of each candidate is shown in Table 2. Because we have four candidates, each node votes points of $3,2,1$, and 0 for four edges. The expected votes are the average of the two candidates if the utilities for two candidates are same. We can see that Edge 1 gets the highest score. For each peripheral node, the edge connecting itself and an isolated node is the first choice, but the edge between the isolated node and the hub is the second choice. An isolated node chooses the candidates that connect itself to other nodes, but has no preference on other candidates. In this way, the candidate between a hub and an isolated node gets the most votes, which makes a hub more connected and stronger.

This kind of phenomenon is visible in some real-world examples. Let us take an airline network for example. Each
Table 3: Expected number of votes with a few nodes.

\begin{tabular}{c|ccccc|c} 
& $\mathrm{a}$ & $\mathrm{b}$ & $\mathrm{c}$ & $\mathrm{d}$ & $\mathrm{e}$ & $\mathrm{Sum}$ \\
\hline initial & $(1.17)$ & $(2.21)$ & $(1.17)$ & $(0.23)$ & $(0.23)$ & \\
Edge 1 & $1(1.52)$ & $0(1.52)$ & $0(1.52)$ & $0(0.23)$ & $1(0.23)$ & 2 \\
Edge 2 & $0(0.85)$ & $1(1.56)$ & $1(1.56)$ & $1(0.85)$ & $0(0.18)$ & 3 \\
\hline \multicolumn{6}{c}{ We show the PageRank values in the parenthesis. }
\end{tabular}

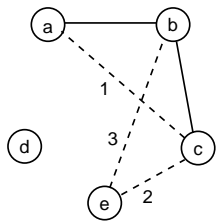

(1)

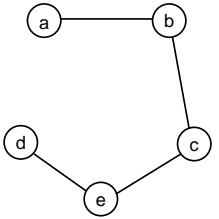

(2)

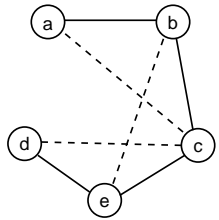

(3)
Figure 10: Illustration of edge addition in case of betweenness centrality.

airline has a few hub airports. Various customers in different regions want to be connected to other cities by shorter distances: they want to increase closeness centrality. Therefore, the emergence of hubs are useful in global situations. In a similar illustrative example, this situation is observed in a supply chain network of various industries. Each company prefers a short chain, thereby encouraging the emergence of a couple of giant companies globally. In this situation, each node come to overly rely on a couple of hubs too much, producing vulnerability against a hub attack, as is known to occur in scale-free networks,

\subsection{Betweenness Centrality}

Two phases pertain in network growth when using betweenness centrality: In the first phase, isolated nodes are connected until all nodes are connected. In this phase, we can assume two types of edge candidate, as depicted in Figure 10(1); 

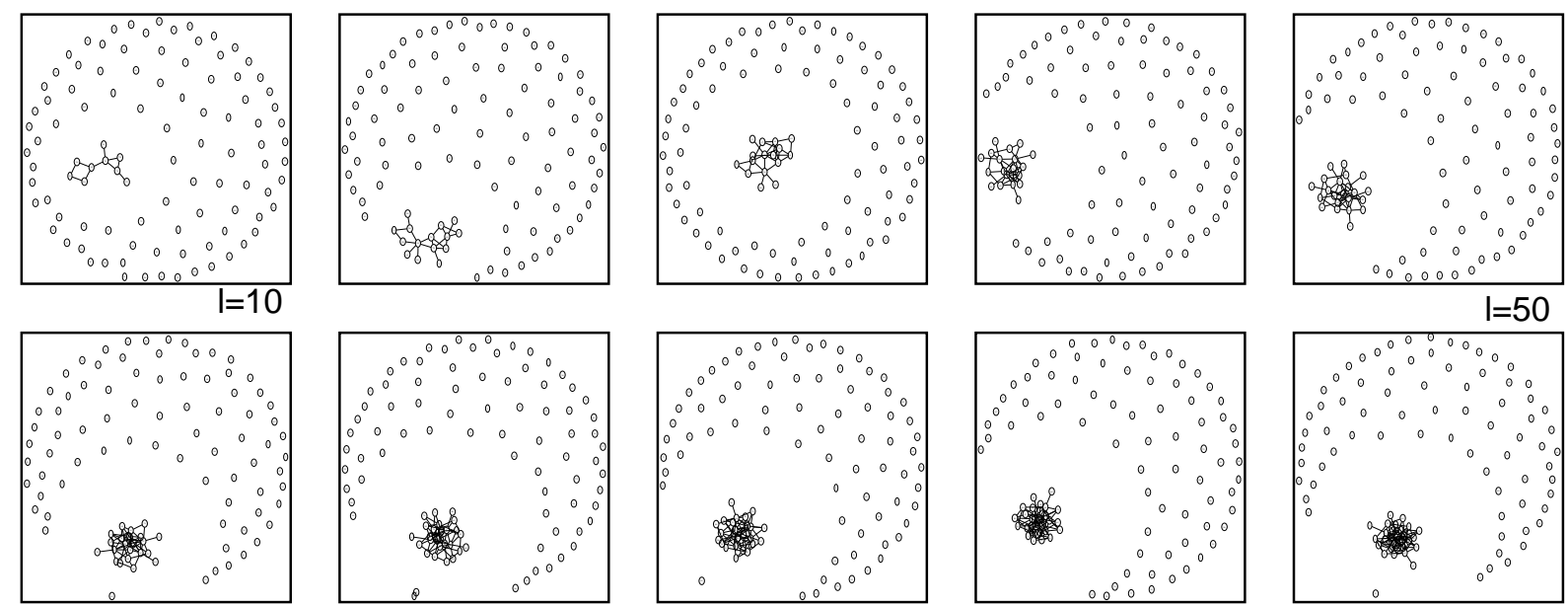

$\mathrm{l}=50$
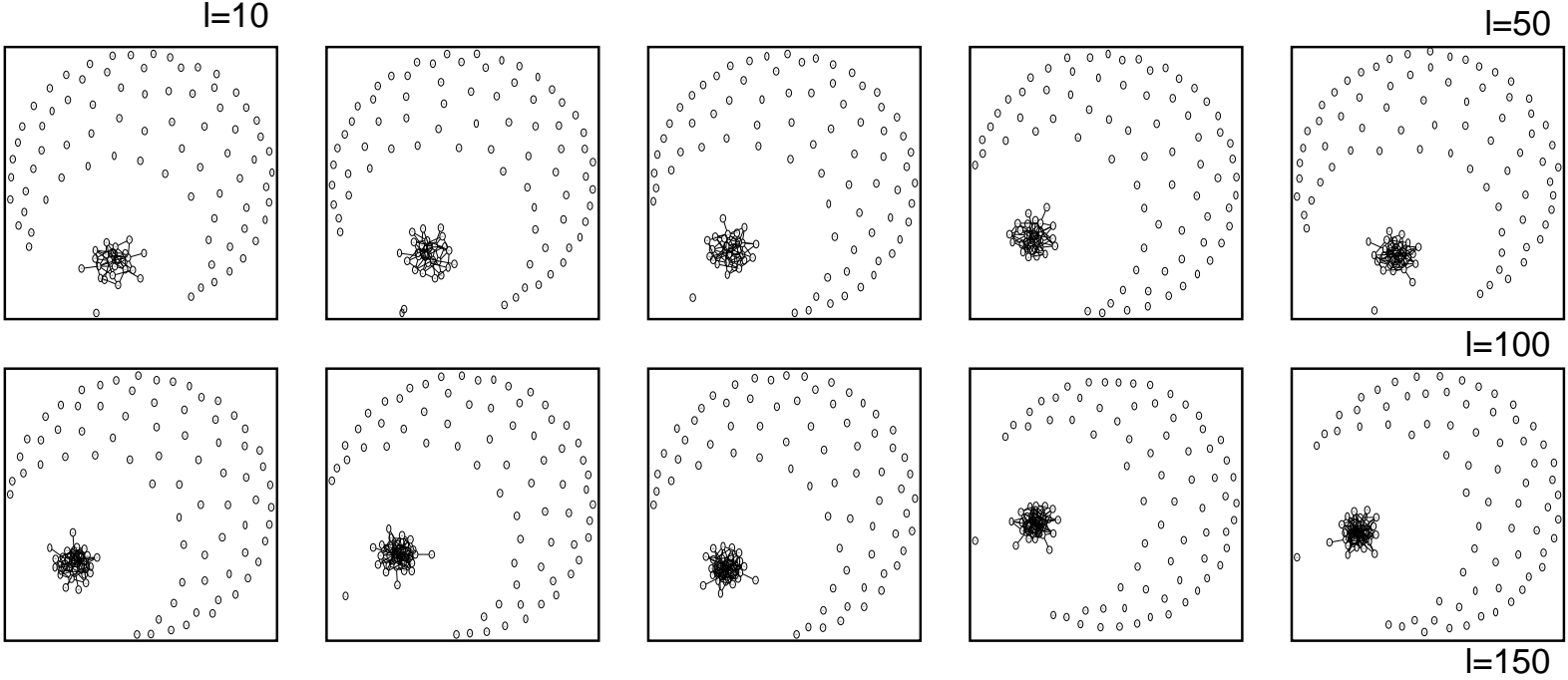

Figure 8: Network growth using PageRank centrality. $N=100, c=200$. Up to 150 edges.

Table 4: Expected number of votes with a large number of nodes.

\begin{tabular}{c|cccccccc|c} 
& $\mathrm{a}$ & $\mathrm{b}$ & $\mathrm{c}$ & $\mathrm{d}$ & $\mathrm{e} 1$ & $\mathrm{e} 2$ & $\ldots$ & $\mathrm{e} 96$ & Sum \\
\hline initial & $(4.39)$ & $(8.32)$ & $(4.39)$ & $(0.84)$ & $(0.85)$ & $(0.85)$ & $\ldots$ & $(0.85)$ & \\
Edge 1 & $1(5.70)$ & $0(5.70)$ & $0(5.70)$ & $0(0.85)$ & $1(0.85)$ & $1(0.85)$ & $\ldots$ & $1(0.85)$ & 97 \\
Edge 2 & $0(3.81)$ & $1(7.06)$ & $1(7.06)$ & $1(3.81)$ & $0(0.82)$ & $0(0.82)$ & $\ldots$ & $0(0.82)$ & 3 \\
\hline
\end{tabular}

Edge 1 An edge within the connected component, and

Edge 2, Edge 3 An edge which connects a connected component and an isolated node.

The former often decrease betweenness of the nodes in the component because the edge will produce shortest paths with less distances, thus smaller number of nodes are included in the shortest paths. Contrarily, the latter always increase betweenness because the existing shortest paths remain and there will be new shortest paths. Comparing Edge 2 and Edge 3, Edge 2 increases the betweenness of Node b and Node c, whereas Edge 3 increases only that of Node b. As a result, Edge 2 gets high score of voting than Edge 3. In this way, a network lengthens and the diameter increases (as in Figure 10(2)).

In the second phase, all the nodes are already connected. No addition of an edge increases betweenness, but which candidate decreases betweenness centrality at least? The best edges on that point are those connecting two nodes with distance of two. We can see those edges in Figure 10(3) as dotted lines. In this way, many will be apparent in the network. When each agents want to be central in terms of information flow (as in bureaucratic organizations) the network tends to have large $L$, sometimes resulting in less efficiency.

\subsection{PageRank Centrality}

We assume simple case first as in Figure 11 to understand the mechanism of PageRank centrality. Two edge types exist:

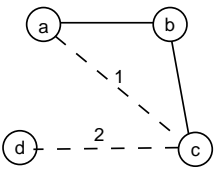

(e)

(1)

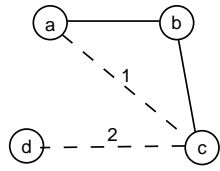

(1) (อ2) ... (996)

(2)
Figure 11: Illustration of edge addition in case of PageRank centrality.

Edge 1 an edge within a connected component, and

Edge 2 an edge which connects a connected component and an isolated node.

Edge 2 is selected where the isolated nodes are few, as in Figure 11(1). Figure 3 shows the expected votes for Edge 1 and Edge2: Edge2 receives more votes. However, in cases with numerous nodes as in Figure 11(2), the situation is different. Edge 1 is selected as in Figure 4. Each isolated node votes for Edge 1 because the decrease of the number of isolated nodes implies the decrease of PageRank value.

As a result, PageRank makes a dense connected component with numerous edges; the number of the connected component increases very slowly. Such a situation is sometimes observed in real-world social networks such as OTAKU communities in Japan. Some edges form a strong group, and other nodes apparently resist connection. The problem is apparently small connectivity among the overall nodes. 


\section{DISCUSSION}

Because we design our model as simple as possible, some important elements were neglected in our model. Those elements should be investigated in the further studies: (i) Edges are monotonously increasing in our model. However, in real-world network, edges can be deleted. Also nodes can be added and deleted. (ii) Centrality measures require knowledge of global network topology (except degree centrality). We can change the model to use the ego-centric centrality measures. (iii) We can mix different agents with different centrality measures and examine the manner in which the network grows. Similarly, we can design a utility function as a weighted sum of several centrality measures and observe the resultant networks. (iv) We employed a Borda count for voting, but we might alternatively use a negotiation model, or some other form to achieve agreements among agents. Actually, we have tried several expansion but we were unable to include their descriptions in this paper because of the space limitation. We shall report those results in future papers.

One application of our model is the analysis of the network. We can infer which centrality measure dominates in an actual social network. For example, we have investigated a social network of academic researchers in Japanese Society for Artificial Intelligence (JSAI) [11], and find that betweenness centrality and closeness centrality are two major ingredients with weights of 0.5 and 0.2 correspondingly. Based on the analysis, we can build a recommendation system in conference support systems [9]: each user agent calculates the utility obtained when the user is connected to other persons. The system makes recommendation to both persons so that they get acquainted if both (and neighboring) agents agree to the possible connection. ${ }^{3}$ Our model is useful in various social systems that employ a multi-agent architecture.

\section{CONCLUSION}

We have proposed an model of the growing network. This model includes a node in a network as an agent that votes based on a utility function. Different centrality measures engender different networks: a scale-free network (closeness), a random graph (degree), a regular graph (betweenness), and a complete graph (PageRank).

We can use our model in various kinds of multi-agent systems, especially of social systems. Actually, the motivation of our research lies in the development of recommendation systems in academic conferences. Although several dimensions that require more investigation in our model, we believe that this research provides helpful insights toward global network properties and local decision from the multiagent perspective.

\section{REFERENCES}

[1] R. Ashri, S. Ramchurn, J. Sabater, M. Luck, and N. Jennings. Trust evaluation through relationship analysis. In Proc. AAMAS 2006, 2006.

[2] R. Axtell. Effects of Interaction Topology and Activation Regime in Several Multi-Agent Systems, pages 33-48. Springer, 2000.

\footnotetext{
${ }^{3}$ From our experience, without such a mechanism, each user agent simply recommends a connection to authoritative persons, which is not appropriate for the community.
}

[3] A.-L. Barabási. LINKED: The New Science of Networks. Perseus Publishing, Cambride, MA, 2002.

[4] A. Birukou, E. Blanzieri, and P. Giorgini. Multi-agent system that facilitates scientific publications search. In Proc. AAMAS 2006, 2006.

[5] U. Brandes. A faster algorithm for betweenness centrality. Journal of Mathematical Sociology, 25(2):163-177, 2001.

[6] S. Brin and L. Page. The anatomy of a large-scale hypertextual web search engine. In Proc. 7 th $W W W$ Conf., 1998.

[7] L. C. Freeman. Centrality in social networks: Conceptual clarification. Social Networks, 1:215-239, 1979.

[8] M. G. Gaston. Agent-organized networks for dynamic team formation. In Proc. AAMAS 2005, 2005.

[9] T. Hope, M. Hamasaki, Y. Matsuo, and T. Nishimura. Strengthening community with embodied social networks. In Proc. HCI2006, 2006.

[10] M. Jackson and A. Watts. The evolution of social and economic networks. Journal of Economic Theory, 106:265-295, 2002.

[11] Y. Matsuo, J. Mori, M. Hamasaki, H. Takeda, T. Nishimura, K. Hasida, and M. Ishizuka. POLYPHONET: An advanced social network extraction system. In Proc. WWW 2006, 2006.

[12] J. Mori, M. Ishizuka, T. Sugiyama, and Y. Matsuo. Real-world oriented information sharing using social networks. In Proc. ACM GROUP'05, 2005.

[13] W. Nejdl, S. Ghita, and R. Paiu. Semantically rich recommendations in social networks for sharing, exchanging and ranking semantic context. In Proc. ISWC 2005, 2005.

[14] A. S. Pentland. Socially aware computation and communication. IEEE Computer, 2005.

[15] J. Pujol, R. Sangüesa, and J. Delgado. Extracting reputation in multi agent systems by means of social network topology. In Proc. AAMAS 2002, 2002.

[16] J. Sabater and C. Sierra. Reputation and social network analysis in multi-agent systems. In Proc. AAMAS 2002, 2002.

[17] S. Staab, P. Domingos, P. Mika, J. Golbeck, L. Ding, T. Finin, A. Joshi, A. Nowak, and R. Vallacher. Social networks applied. IEEE Intelligent Systems, pages 80-93, 2005.

[18] W. Wang, B. Hu, T. Zhou, B. Wang, and Y. Xie. Mutual selection model for weighted networks. Physical Review E, 72, 2005.

[19] S. Wasserman and K. Faust. Social network analysis. Methods and Applications. Cambridge University Press, Cambridge, 1994.

[20] D. Watts. Six Degrees: The Science of a Connected Age. W. W. Norton \& Company, 2003.

[21] B. Wellman. The global village: Internet and community. The Arts $\&$ Science Review, University of Toronto, 1(1):26-30, 2006.

[22] J. Weng, C. Miao, and A. Goh. Trust-based agent community for collaborative recommendation. In Proc. AAMAS 2006, 2006. 